\title{
ON MODELING OF TRANSITIONAL FLOW AND ITS APPLICATION ON A HIGH LIFT MULTI-ELEMENT AIRFOIL CONFIGURATION
}

\author{
Andreas Krumbein*, German Aerospace Center (DLR), Braunschweig, Germany
}

\begin{abstract}
$\underline{\text { Abstract }}$
In order to enhace its capabilities to handle flows with transition a RANS solver has been extended with regard to the modeling of transitional flow regions based on transition length models and the intermittency function. As the full coupling of the solver to an $\mathrm{e}^{N}$-method that predicts the locations of transition onset has not yet been completed the points of laminar separation are supposed to represent the transition locations in a first step. A method and an algorithm for detecting the laminar separation points are derived, the intermittency function and two transition length models are implemented and validated at a selected high lift multi-element test case. The paper focuses on the background of the implementation work and the testing of the functionalities of the algorithms. Details of the implementation, which are consequences of an underlying transition prediction strategy are outlined. The testing is described and documented by a number of commented plots.
\end{abstract}

\section{Introduction}

The modeling of laminar-turbulent transition in Reynolds-averaged Navier-Sokes solvers (RANS) is a necessary requirement for the computation of airfoils and wings in aerospace industry, as it is not possible to obtain quantitatively correct results, if the laminar-turbulent transition is not taken into account. For the design process of a wing in aerospace industry exists the demand for a RANS-based CFD tool that is able to automatically and autonomously handle flows with laminarturbulent transition.

First steps towards the setup of such a tool were made e.g. in ${ }^{1}$, where a RANS solver and an $\mathrm{e}^{N}$-method ${ }^{2-3}$ based on linear stability theory and the parallel flow assumption, were applied or in ${ }^{4}$, where a RANS solver, a laminar boundary layer method ${ }^{5}$ and an $\mathrm{e}^{N}$-method were coupled. There, the boundary layer method was used to produce highly accurate laminar, viscous layer data to be analyzed by a linear stability code. Such, the very expensive grid adaptation necessary to produce accurate viscous layer data directly from the NavierStokes grid was avoided. The use of an $\mathrm{e}^{N}$-database method $^{6}$ results in a coupled program system that is able to automatically handle transition prediction. Alternative approaches using a transition closure model or a transition/turbulence model directly incorporated into the RANS solver are documented in ${ }^{7-9}$.

At the German Aerospace Center (DLR) the structured RANS code FLOWer ${ }^{10}$ is used together with the laminar boundary layer method ${ }^{5}$ and the $\mathrm{e}^{N}$-database

${ }^{*}$ Research Scientist

Copyright $\odot 2002$ by EU Project HiAer. Published by the American Institute of Aeronautics and Astronautics, Inc., with permission. method $^{6}$. The laminar boundary layer method and the $\mathrm{e}^{N}$-database method are forming a so called 'transition prediction module' that is coupled to the RANS solver and that interacts with the RANS solver during the computation ${ }^{11-12}$. Presently, the transition prediction module of FLOWer can be applied to 2-dimensional one-element configurations.

The description of transitional flow regions in FLOWer is done by the application of point transition, which means that turbulence quantities which are supressed in the laminar part of the flow suddenly become active at the location of transition onset. This procedure results in a sudden change of the flow quantities around the location of transition onset. Although due to the effects of numerical dissipation a small transitional-like flow region is generated artificially, the sudden change of the flow quantities is often strong enough to prevent the iterative transition prediction process to converge ${ }^{13}$. In addition, the application of point transition comes along with a strong upstream influence such that the transitional-like flow region starts considerably upstream of the transition location. In 2-dimensional airfoil flows an upstream influence up to $10 \%$ of the chord length of the airfoil can be observed.

The extension of the FLOWer code to avoid these two drawbacks - the restricted application of the transition prediction module to 2-dimensional one-element configurations and the application of point transition instead of the physical modeling of transitional flow - is currently under way. The work is done in the European project HiAer - High Level Modelling of High Lift Aerodynamics ${ }^{14}$. In the HiAer project, the coupled program system is extended to 2-dimensional multi-element configurations and physical models for the computation of transitional flow regions are introduced. The extended code is applied to 2-dimensional high lift systems.

The extensions are performed in two steps. First, a generalized infrastructure in the FLOWer code with respect to the transition prediction module is build up and tested. I.e., the code is changed in such a way that the transition prediction module can be activated in the future for arbitrary multi-element configurations independent of the block topology and the grid structure. Second, the transition prediction module is coupled to the generalized infrastructure.

In the framework of this paper, the first extension step is documented. Thus, this paper has the character of a progress report.

As up to now the transition prediction module is not yet coupled to the extended FLOWer code, the locations of 
laminar separation determined by the FLOWer code are supposed to represent the laminar-turbulent transition locations in a first step. In many cases, this assumption leads to a good approximation of the real transition point. This may be the case for low Reynolds number airfoil flows when transition does not occur before the laminar boundary layer separates.

As the term 'transition prediction' in this restricted context is not accurate the verbalization 'transition determination' is used for the applied handling of points where transition is fixed throughout this paper.

The main objective of the perfomed work presented in this paper is to supply a reliably working infrastructure in a RANS code such that the RANS code together with the transition prediction module described above can be used in the future for the computation of 2-dimensional multi-element high lift systems of aircrafts including transitional flow regions. In order to achieve the new objectives in the FLOWer code the steps which have to be done according to the first extension step are:

-Implementing the capability to fix transition at the point of laminar separation in the RANS computation

-Implementing the capability to compute transitional flow regions

These two issues are the subjects of this paper.

A method and an algorithm for detecting the laminar separation points are derived and implemented into the FLOWer code. For each element of a high lift configuration on the upper and lower side the laminar separation point will be detected and the transition fixed there. The intermittency function and two transition length models are implemented and validated in a variety of test computations at a selected high lift multi-element test case.

This paper focuses on the background of the implementation work and the testing of the functionalities of the algorithms. Details of the implementation, which are consequences of the underlying transition prediction strategy 4-6, 11-12 are outlined. The testing is described and documented by a number of commented plots of the results of the transition determination procedure and of the transition length models.

\section{Implementation}

FLOWer is a 3-dimensional, compressible RANS code for steady or unsteady flow problems and uses structured body-fitted multi-block-meshes. The code is based on a finite volume method and a cell vertex spatial discretization scheme and uses an explicit Runge-Kutta time integration scheme with multi-grid acceleration. The influence of turbulence is taken into account by eddy viscosity turbulence models according to the Boussinesq approximation.
The implementations are independent of the block topology of the computational grid and of the grid structure (structured, unstructured or hybrid grid).

For the implementations necessary there are three thematically different areas: the handling of the surface points of the configuration in order to build up a method which is independent of the block topology and the grid structure, the detection of laminar separation and the generation of transitional flow regions in the code.

\subsection{Handling of Surface Points}

The complete coupled program system that will be used for transition prediction with the RANS solver FLOWer consists of the RANS solver itself ${ }^{10}$, a laminar boundary layer method for swept, tapered wings ${ }^{5}$ and a transition prediction method, which is provided with all necessary data, e.g. boundary layer parameters, by the laminar boundary layer method. Besides a number of empirical transition criteria, the most general transition prediction method that is available in the FLOWer transition prediction module is an an $\mathrm{e}^{N}$-database method ${ }^{6}$.

The RANS solver communicates the surface pressure distribution of the configuration as input data to the laminar boundary layer method, the laminar boundary layer method computes all the boundary layer parameters that are needed for the transition prediction method, the transition prediction method determines new transition locations that are given back to the RANS solver. This coupling structure ends up in an iteration procedure for the transition locations within the iteration of the RANS equations.

As a boundary layer method is an essential part of the coupled program system there is a number of conditions which must be fulfilled by the way of handling the surface points of the configuration during the iteration process:

-Each element of a multi-element configuration must be divided into an upper and a lower side. The point which defines the division is the stagnation point on the airfoil surface.

-The surface points on upper and lower side must be ordered. The sequences of points start at the stagnation point and end at the trailing edge points of upper side or lower side respectively.

-The ordered sequences of points must not contain topologically singular surface grid points. This may happen in the case that the surface of an airfoil is contained in more than one block. The surface points on the block cuts are topologically non-unique. Each physical surface point must exist only once in the ordered sequence of points.

To fulfill these conditions the following steps can be done: 
-The identification values of the surface points of each element are stored in an additional array. As these identification values are integer values - the i-index, j-index and k-index and the block number in case of a structured solver or the point number in case of an unstructured solver - integer arrays are sufficient for this purpose. All the following steps are done using these new arrays. The identification values in the new arrays are used to adress the surface grid points and to have access to the flow variables at the surface points.

- Together with the additional arrays for the identification values one needs another integer array for individual information about each surface point of each element. This array contains information about the single surface point and the ordered sequence of the surface points. This array is called $U L F L G$ in this paper. $U L F L G$ stands for 'Upper-Lower-Flag' as it contains the 'upper-lower' status of the surface point.

Let $P_{S}\left(n_{e}\right)$ be a surface point of element $n_{e}, 1 \leq n_{e} \leq N_{e}$, $N_{e}$ being the maximum number of configuration elements and $\operatorname{ULFLG}\left(P_{S}\left(n_{e}\right)\right)$ its 'Upper-Lower-Flag'.

First, the 'Upper-Lower-Flag' for each surface point is initialized,

$$
\operatorname{ULFLG}\left(P_{S}\left(n_{e}\right)\right)^{\text {init }}=7 \text {, }
$$

the value ' 7 ' being just an arbitrary value for the intialization.

-When the run of the RANS code starts, during the initialization phase, an a priori division of each element into upper and lower side is performed. The division is defined by the geometric nose point of each element and by the rearmost trailing edge point of the element. The rearmost trailing edge point is the one that has the greatest co-ordinate value with respect to the chordwise direction of the element. The geometric nose point is the surface point that has the greatest distance from the rearmost trailing edge point.

The division into upper and lower side is easy for airfoils with two trailing edges. In this case, the user can give in the co-ordinates of two points which define a straight line that devides the airfoil into upper and lower side. One point may be any point on a line between upper and lower trailing edge, the other point may be near the geometric nose point, for example, or the geometric nose point itself.

For airfoils with one trailing edge the division should be done automatically, a procedure that can be applied for airfoils with two trailing edges too, of course. In this case, it is necessary to approximate the mean line of the airfoil. As a very weak condition for the quality of the approximation of the mean line it must be ensured that all the points of the polygonial line that defines the mean line are located within the airfoil contour.
The geometric nose point belongs to both sides. For this point it is set

$$
\operatorname{ULFLG}\left(P_{S, \text { nose }}\left(n_{e}\right)\right)=0 \text {. }
$$

A surface point on the upper side of the airfoil gets

$$
\operatorname{ULFLG}\left(P_{S, u p p}\left(n_{e}\right)\right)=1 \text {, }
$$

a surface point on the lower side of the airfoil gets

$$
\operatorname{ULFLG}\left(P_{S, \text { low }}\left(n_{e}\right)\right)=-1 \text {. }
$$

After this step there exists an geometrical division of each element into upper and lower side.

-All pairs of points which are topologically non-unique are identified. One point of each pair and all trailing edge points get

$$
\operatorname{ULFLG}\left(P_{S, n u}\left(n_{e}\right)\right)=2 *^{\operatorname{sgn}}\left[\operatorname{ULFLG}\left(P_{S, n u}\left(n_{e}\right)\right)\right] \text {. }
$$

During the transition determination procedure surface points with

$$
\left|\operatorname{ULFLG}\left(P_{S}\left(n_{e}\right)\right)\right|=2
$$

are not taken into account.

-For each element the surface points first on the upper side and then on the lower side are ordered according to its Euclidian distance from the geometric nose point. The ordering is performed within the array $\operatorname{ULFLG}\left(n_{e}\right)$. After this step, the surface points of upper and lower side of each element are in an ordered sequence along the airfoil contour from a geometrical point of view.

-During the transient phase of the RANS computation the stagnation point is determined. The stagnation point defines the aerodynamical division of each airfoil into upper and lower side and its location changes the number of points which belong either to upper or lower side. Everytime when the stagnation point has been determined the corresponding surface point gets

$$
\operatorname{ULFLG}\left(P_{S, \text { stag }}\left(n_{e}\right)\right)=0 \text {. }
$$

The stagnation point has moved either into the area of the former upper side or into the area of the former lower side. For all the surface points that are located between the two points which are marked with $\operatorname{ULFLG}\left(P_{S}\left(n_{e}\right)\right)=0$ the algebraic sign must be reversed and the 'Upper-Lower-Flag' of the 'old' stagnation point must be given the correct value. In the case that the 'new' stagnation point has moved into the area of the former lower side

$$
\operatorname{ULFLG}\left(P_{S, \text { stag }}\left(n_{e}\right)\right)^{\text {old }}=1
$$

is set, in the case that the 'new' stagnation point has moved into the area of the former upper side

$$
\operatorname{ULFLG}\left(P_{S, \text { stag }}\left(n_{e}\right)\right)^{\text {old }}=-1
$$

is set.

-Finally, for each element the surface points between the 'old' and the 'new' stagantion point are ordered according to its arc length along the airfoil contour measured from the 'new' stagnation point. The ordering is again performed within the array $\operatorname{ULFLG}\left(n_{e}\right)$. After this 
step, the surface points of upper and lower side of each element are in an ordered sequence along the airfoil contour from an aerodynamical point of view.

\subsection{Detection of Laminar Separation}

The algorithm for the detection of laminar separation consists of two parts, the determination of the stagnation point and the determination of separation points.

The algorithm itself does not make a distinction between laminar and non-laminar separation points, it simply detects separation points. But from the global strategy for the transition determination iteration it is clear that always when the algorithm is interpreting a separation point as a transition point it must be a laminar separation point. This is the case only when a separation point is located upstream of the actual transition point, i.e. the transition point that is currently dividing the airfoil side into a laminar and a non-laminar part at this stage of the transition location iteration.

The stagnation point is defined as the surface grid point where the maximum $c_{p}$-value is found, and it holds

$$
c_{P}\left(P_{S, \text { stag }}\left(n_{e}\right)\right)=\max _{P_{S}}\left[c_{P}\left(P_{S}\left(n_{e}\right)\right)\right] .
$$

After the division of the current airfoil into upper and lower side has been done, the algorithm is looking for a separation point, first on the upper then on the lower side. The search is starting at the stagnation point for each side of the airfoil and ends at the corresponding trailing edge point. The existance of a separation point $\boldsymbol{r}_{\text {sep }}$ is definded by the following condition with the position counter $i$, that counts the surface points from the stagnation point to the trailing edge point, the tangential velocity vector $v_{t}$ at the first grid point $P^{(1)}$ apart from the solid wall of the airfoil, the direction vector $\Delta r_{i+1, i}$ pointing from point $P^{(1)}{ }_{i+1}$ to point $P^{(1)}, \Delta r_{i+1, i}$ $=\boldsymbol{r}\left(P^{(1)}{ }_{i+1}\right)-\boldsymbol{r}\left(P^{(1)}{ }_{i}\right)$ and '• ' indicating the scalar product between vectors:

$$
\operatorname{sgn}\left[v_{t}\left(P^{(1)}{ }_{i}\right) \bullet \Delta r_{i+1, i}\right]=\operatorname{sgn}\left[v_{t}\left(P^{(1)}{ }_{i+1}\right) \bullet \Delta r_{i+1, i}\right]
$$

$\Rightarrow$ It does not exist a separation point $\boldsymbol{r}_{\text {sep }}$ in the closed interval between the surface points $P_{S, i}$ and $P_{S, i+1}$.

$$
\operatorname{sgn}\left[v_{t}\left(P^{(1)}{ }_{i}\right) \cdot \Delta r_{i+1, i}\right] \neq \operatorname{sgn}\left[v_{t}\left(P^{(1)}{ }_{i+1}\right) \bullet \Delta r_{i+1, i}\right] \text { (13) }
$$

$\Rightarrow$ It exists a separation point $\boldsymbol{r}_{\text {sep }}$ in the closed interval between the surface points $P_{S, i}$ and $P_{S, i+1}$.

In the case that the detected separation point $\boldsymbol{r}_{\text {sep }}$ is located upstream of the transition point currently used, $\boldsymbol{r}_{\text {sep }}$ is a laminar separation point and surface point $P_{S, i}$ is used as new transition location on the corresponding side of the airfoil.

\subsection{Generation of Transitional Flow Regions}

In the case that a new transition location has been determined the laminar, transitional and turbulent flow regions must be generated anew within the computational grid.
The generation of the different regions is done by the setting of a real value flag $\mathrm{flg}_{\mathrm{glt}}$ at each point of the computational grid that is multiplied with the value of the eddy viscosity $\mu_{t}$ which is computed for every point in the flow field. $f g_{l t}$ is applied in the following way for all the points on solid walls of the configuration,

$$
\mu_{t}^{\text {code }}\left(P_{S}\right)=f \lg _{l t}\left(P_{S}\right) \mu_{t}\left(P_{S}\right) \text {, }
$$

with $f g_{l t}\left(P_{S}\right)=0.0$ for a laminar surface point, $f_{l_{l t}}\left(P_{S}\right)$ $=1.0$ for a turbulent surface point and $f \lg _{l t}\left(P_{S}\right)=\gamma\left(P_{S}\right)$ for a transitional surface point, $\gamma\left(P_{S}\right)$ being the value of the intermittency function $\gamma$ at surface point $P_{S}$.

The laminar length on upper or lower side of an airfoil is defined by the interval between the stagnation point and the transition point on the side $q$, with $q=u, l$ indicating either the upper or the lower side of the airfoil, $0 \leq s_{q} \leq s_{q, t r}{ }^{\text {beg }}, s_{q}$ being the arc length on the side $q$ starting at the stagnation point. The turbulent length is defined by the interval between the ending point of the transitional region and the trailing edge point on side $q$, $s_{q, t r}^{\text {end }} \leq s_{q} \leq s_{q}^{\text {trail }}$, and the transitional length is the interval between the transition point and the ending point of the transitional region, $s_{q, t r}$ beg $<s_{q}<s_{q, t r}$ end . The different intervals are depicted in fig.1.

$\gamma$ is expressed as

$$
\begin{aligned}
& \gamma(x)=1-\exp \left(-0.412 \xi^{2}\right), \\
& \text { with } \\
& \xi=\left(x-x_{t r}{ }^{b e g}\right) / \lambda
\end{aligned}
$$

according to ${ }^{15}, x$ being the longitudinal co-cordinate of a flat plate with its origin located in the upstream end of the plate and $\lambda$ being a measure of the extent of the transitional region. According to ${ }^{13}$, the ending point of the transitional region $x_{t r}^{\text {end }}$ can be defined as

$$
x_{t r}{ }^{\text {end }}=x(\gamma=0.99)
$$

which yields

$$
\lambda=\left(x_{t r}{ }^{\text {end }}-x_{t r}{ }^{\text {beg }}\right) / 3.36 \text {. }
$$

For the determination of the extent of the transitional region $\Delta x_{t r}=x_{t r}{ }^{e n d}-x_{t r}{ }^{b e g}$ the formulas from ${ }^{16}$,

$$
R e_{\Delta x_{t r}}=5.2\left(R e_{x_{t r} \text { beg }}\right)^{3 / 4}
$$

for flows without pressure gradient, and

$$
R e_{\Delta x_{t r}}=2.3\left(R e_{\delta_{t r}^{*} \text { beg }}\right)^{3 / 2}
$$

for flows with pressure gradient, are applied, as it is recommended in ${ }^{13}$ for flows in which transition does not occur before laminar separation, which is the case for all computations whose results are presented in this paper due to the underlying way of determining the transition points by fixing transition at the locations of laminar separation. In these formulas $R e$ is the Reynolds number and $\delta^{*}=\delta^{*}(x)$ the displacement thickness,

$$
\delta^{*}(x)=\int_{0}^{\delta(x)}\left(1-\frac{(\rho \mathrm{U})(\mathrm{y})}{\rho_{\mathrm{e}} \mathrm{U}_{\mathrm{e}}}\right) \mathrm{dy} .
$$


The thickness of the laminar boundary layer $\delta$ was evaluated according to a procedure described in ${ }^{4}$. Thus, $\delta^{*}$ and $\rho_{e}$ and $U_{e}$, the values of the density and the tangential flow velocity at the boundary layer edge, could be determined.

For the application in the RANS solver the $x$ co-ordinate in the formulas is replaced by the arc length $s, s_{q, t r}{ }^{b e g}$ is given by the location of a laminar separation point and $s_{q, t r}$ end is determined by formula (a), based on equation (20), or formula (b), based on equation (19), respectively. Formula (a) reads

$$
s_{q, t r}{ }^{e n d}(a)=2.3 \sqrt{\left(\frac{U_{e}}{v_{e}}\right)_{s_{q, t r}} \text { beg }}\left(\delta_{s_{q, \text { beg }}}^{*}\right)^{\frac{3}{2}}+s_{q, t r} \text { beg },
$$

formula (b) reads

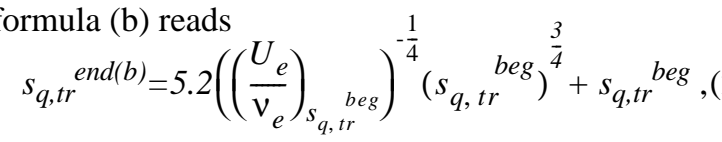

and the intermittency function $\gamma$ is applied in the form

$$
\gamma\left(s_{q}\right)=1-\exp \left(-0.412\left(3.36 \frac{s_{q}-s_{q, t r} \text { beg }}{s_{q, t r} e^{n d}-s_{q, t r} b e g}\right)^{2}\right)
$$

The compuational tests will show that only formula (b), based on flat plate theory, will yield results which compare well enough with experimental findings.

After all the surface points on upper and lower side of an airfoil have been assigned to either the corresponding laminar, turbulent or transitional interval, the field points - all points apart from the solid walls - are treated in the following way:

Within a limiting wall normal distance that can be adjusted by the user of the code every field $P_{F}$ point assumes the flag value of the surface point $P_{S}{ }^{n s t}$ that is located nearest to $P_{F}$,

$$
f l g_{l t}\left(P_{F}\right)=f l g_{l t}\left(P_{S}^{n s t}\left(P_{F}\right)\right) \text {. }
$$

By this treatment, a laminar and a transitional zone for the current element is generated within a turbulent rest of the computational grid. A partitioning into a pure laminar zone within a turbulent rest of the flow domain (point transition) is shown in fig.2. All the steps of this procedure must be applied subsequently to all elements $n_{e}, 1 \leq n_{e} \leq N_{e}$, of the configuration for which transition determination is performed. The order of the elements within the procedure is irrelevant.

\subsection{Test Case}

\section{$\underline{3 \text { Computations }}$}

The test case used to investigate the functionality of the algorithms is the 2-dimensional A310 3-element landing configuration consisting of slat, main airfoil and flap ${ }^{17}$. The turbulence model used is the Spalart-Allmaras 1equation transport model with Edwards modification and the following different computational cases have been performed:
- fully turbulent

- prescribed (fixed) point transition

- determined transition, point transition

- determined transition, transition length (a)

- determined transition, transition length (b)

\subsection{Results}

Fig. 3 shows the convergence history of the computations of cases $\{1\},\{2\},\{3\}$ and $\{4\}$ (a). For all computations with transition a 3-level multi grid method was applied, the fully turbulent computation had to be run in single grid mode. All computations with transition converge satisfactorily fast. For the runs with transition determination the flow field was initalized with the solution of the fully turbulent computation after 15,000 RANS cycles. The fully turbulent computation needs about 70,000 cycles to converge. The attempt of a preconditioned computation did not succeed as the density residual leveled out at an order of magnitude of $10^{-2}$.

In this context, a computation is considered to be converged in the case that the value of the lift coefficient $c_{1}$ does not change anymore at least before the $4^{\text {th }}$ decimal digit and the value of the drag coefficient $c_{d}$ does not change anymore at least before the $6^{\text {th }}$ decimal digit.

Fig. 4 and fig.5 show the convergence history of the transition locations applying point transition and formula (a). For all three elements of the configuration, the longitudinal co-ordinates of the separation locations are plotted versus the RANS iteration cycles. Laminar and non-laminar separation locations were plotted. The laminar separation locations are marked with square symbols, the non-laminar separation locations are not marked. All laminar separation points have been set as transition locations on the upper sides of the elements.

The procedure starts with initially set transition locations at the trailing edges of all elements. During the computation the laminar separation points are moving from the trailing edges towards the nose of each element. Because of the high angle of attack this happens on the upper sides only.

In both cases, the laminar separation stops near the element's nose on all three elements. In the case of point transition, the non-laminar separation vanishes on slat and main airfoil and moves back the trailing edge of the flap. In the case of formula (a), a non-laminar separation point remains directly downstream of the transition point of the main airfoil and the flap. On the slat, the non-laminar separation vanishes after about 1600 RANS cycles.

The transition locations which existed during the experimental measuring of the configuration are plotted as black circular symbols on the surface of the elements. The transition points on slat and flap have been determined using an approximate computational transition pre- 
diction method. The transition point on the main airfoil is the position of a transition band which tripped the bounday layer in the experiment. The differences $\Delta x_{t r}$ between the 'experimental' and the computed values of the transition locations are given as

$$
\Delta x_{t r}=x_{t r}{ }^{\text {comp }}-x_{t r}{ }^{\text {exp }}
$$

in the figures. For the locations of free transition on slat and flap both cases yield very good results.

In the next figures, the convergence histories of the transition locations are shown in a blow up for the main airfoil, fig.6-7, and the flap, fig.8-9 with the corresponding surface friction distributions $c_{f}$. The regions on the upper sides of the elements which are marked with a black line indicate the domains in which the separation locations - laminar and non-laminar - are moving to and fro. In fig.7 and 9 the non-laminar separation regions downstream of the transition points are clearly visible on main airfoil and flap.

Fig. 10 and 11 show the distributions of $f \lg _{l t}$ along the upper sides of the surface contours of all elements and thus the computed transitions lengths, $l_{\text {trelem }}$, resulting from formula (a) and formula (b). Formula (a) yields values between $12 \%$ and $15 \%$ of the corresponding element's chord length - as given in the figures -, which are much greater compared to experimental findings in which values between $3 \%$ and $5 \%$ are usual. Formula (b), however, yields the expected magnitude of the values of the transition lengths and prevents the transitional separations directly downstream of the transition locations.

Fig.12 compares the convergence behaviour of the determination procedure applying the two different formulas and shows that the final values of the transition locations are the same.

Fig.13 shows the $c_{p}$-distributions for all cases, fig.14 shows the $c_{f}$-distributions for the cases $\{1\},\{2\},\{3\}$ and $\{4\}$ (a) and fig. 15 compares the $c_{f}$-distributions for the cases $\{4\}$ (a) and $\{4\}(b)$.

As expected, the pressure distributions between the fully turbulent case on the one hand and the cases with transition on the other hand show a clearly visible gap on the upper sides of each element of the configuration. The width of the gap has its greatest extent in the suction zones near the noses of the elements. The pressure distributions of all the cases with transition can not be distinguished in this representation.

Comparing the $c_{f}$-distributions of the main airfoil of cases $\{2\}$ and $\{3\}$ in fig. 14 one can clearly see the very strong upstream influence of case $\{3\}$. The location of the local minimum friction value which marks the end of the laminar boundary layer is almost the same as in case $\{2\}$, although in case $\{3\}$ the location of transition onset is located much more downstream than in case
$\{2\}$. The difference between the 'numerically' simulated transitional region in case $\{3\}$ (upstream influence) and the physically simulated transitional region in case $\{4\}$ is remarkably. The physically simulated transitional region is almost as twice as long as the computed transitional length given by the model.

In fig. 15 one can clearly see the difference caused by the different transition length models. For formula (b), the transitional separations on main airfoil and flap directly downstream the transition points that existed for formula (a) do not appear.

In the table following below, a summary of the computed force coefficients is given. The differences between two force coefficients $c_{k, 1}$ and $c_{k, 2}$ were determined according to

$$
\Delta c_{k}=\left(c_{k, 2}-c_{k, 1}\right) / c_{k, 1}, k=l, d
$$

and give an impression of the integral effect of the different modelling levels. The values $c_{k, 1}$ and $c_{k, 2}$ are taken from two consecutive lines of the table, where the index ' 1 ' indicates the upper line and the index ' 2 ' the lower

\begin{tabular}{|c|c|c|c|c|}
\hline & $c_{l}$ & $c_{d}$ & $\Delta c_{l}$ & $\Delta c_{d}$ \\
\hline $\begin{array}{l}\text { fully } \\
\text { turbulent, } \mathrm{ft}\end{array}$ &. & $\begin{array}{c}. \\
\text { counts }\end{array}$ & $4.5 \%$ & $-10 \%$ \\
\hline $\begin{array}{l}\text { trans, fixed, } \\
\text { point }\end{array}$ & $\begin{array}{c}c_{l}^{f t}+ \\
0.1953\end{array}$ & $\begin{array}{l}c_{d} f^{t}- \\
130.5\end{array}$ & \multirow[t]{2}{*}{$0.4 \%$} & \multirow[t]{2}{*}{$-0.7 \%$} \\
\hline trans, det., & $c_{l}^{f t}+$ & $c_{d}^{f t}-$ & & \\
\hline point & 0.2131 & 139.2 & \multirow{2}{*}{$0.13 \%$} & \multirow{2}{*}{$-0.08 \%$} \\
\hline $\begin{array}{l}\text { trans, det., } \\
\text { formula (a) }\end{array}$ & $\begin{array}{c}c_{l}^{f t}+ \\
0.2191\end{array}$ & $\begin{array}{l}c_{d}^{f t}- \\
140.2\end{array}$ & & \\
\hline $\begin{array}{l}\text { trans, det., } \\
\text { formula (b) }\end{array}$ & $\begin{array}{c}c_{l}^{f t}+ \\
0.2174\end{array}$ & $\begin{array}{l}c_{d}^{f t}- \\
140.6\end{array}$ & $-0.04 \%$ & $-0.03 \%$ \\
\hline
\end{tabular}
line.

\section{$\underline{3.3 \text { Initialization }}$}

A basic idea for the transition determination process is to start the flow computation with a flow field initialized with free stream values and transition locations set very far downstream, e.g. at the trailing edges, such that, effectively, a fully laminar flow is computed in the beginning of the flow computation. During the transient phase of the computation the successively detected laminar separation points are then used as transition locations until a converged state of the transition locations has been reached. However, the strategy to start the computation with free stream values fails because of the following reason:

For the detection of separation points on either the upper or the lower side of an airfoil the location of the stagnation point on the airfoil must be known, as it divides the 
upper from the lower side from the aerodynamical point of view. The stagnation point is the point of $c_{p, \max }$ at the airfoil surface. Additionally, it is a point where the tangential velocity vectors of two differentially neighouring points have different algebraic signs. In a steady flow these two facts are valid for the same physical point in space.

A separation point is characterized by two differentially neighouring points whose tangential velocity vectors have different algebraic signs, too.

In the first tests with airfoil flows under high angles of attack it turned out that the two conditions that define the stagnation point do not indicate the same point in space during the transient phase of the computation. Usually, the point with $c_{p, \max }$ is very near the 'real' stagnation point from the very beginning throuhgout the transient phase until the RANS computation has converged to a steady solution.

The point whose neighours have different signs of their tangential velocity vectors is, at first, located very near to the geometric nose point of the airfoil. During the transient phase it is moving downstream from the geometric nose point towards the point with $c_{p, \max }$. When the RANS computation has converged these two points have become one single point, the stagnation point.

Thus, during the transient phase it is not possible to use these two conditions to detect the stagnation point. Only the $c_{p, \max }$ criterion is usable to find the stagnation point. As also a separation point is characterized by two differentially neighouring points whose tangential velocity vectors have different algebraic signs the first detected separation point is the 'wrong stagnation point' that is moving towards the point with $c_{p, \max }$.

As a consequence, it does not seem possible to start the computation using free stream values as initial values. To overcome this problem the computation may be initialized with a converged steady solution of a fully turbulent flow field. In this case, the two conditions which define the stagnation point mark the same physical point in space. All computations initialized with a fully turbulent flow field yielded very good results.

Fig.16 and 17 give an impression of the computations which were initialized with free stream values. When the separation points are determined for the first time, after about 120 RANS cycles, the "wrong stagnation point' on the main airfoil is detected as a laminar separation point, located very near to the nose of the main airfoil, and is set as transition point. The computations result in a strongly detached flow over main airfoil and flap. Also in the cove of the slat, the flow is fully detached. This behaviour is remarkable as the flow over the main airfoil is fully turbulent on the complete upper side of the airfoil, starting at the 'wrong stagnation point', streaming along the nose up to the upper trailing edge.
In the two figures the position of the 'wrong stagnation point' on the main airfoil is marked by an arrow. From this point on, the flow is fully turbulent on the upper side and fully laminar on the lower side. The coloured lines show again the separation points plotted versus the RANS iteration cycles.

\subsection{Other Turbulence Models}

In addition to the application of the Spalart-Allmaras model with Edwards modification other turbulence models were applied to test the strategy of the transition determination procedure for the selected test case. The selected models are the algebraic Baldwin-Lomax model, the standard Wilcox k- $\omega$ model and the LEA k- $\omega$ model. The LEA $k-\omega$ model is the linear body of an explicit algebraic Reynolds stress model based on the standard Wilcox $\mathrm{k}-\omega$ model as background model. The LEA $\mathrm{k}-\omega$ model uses

$$
\mu_{t}=c_{\mu} \rho k / \omega
$$

to compute the eddy viscosity $\mu_{t}$ with $c_{\mu}$ being a function of some of the local flow field variables, whereas in the standard Wilcox k- $\omega$ model $c_{\mu}=1$.

For the computations using these turbulence models the same parameter settings and the same strategy as before were applied. In the computations with determined transition locations only formula (b) was applied. All the computations had to be carried out in single grid mode as for multigrid computations no convergence could be obtained. For all computations using transport equation turbulence models the fully implicit treatment of the turbulence equations (DDADI scheme) was applied.

As expected, the convergence behaviour for the computations using the Baldwin-Lomax turbulence model is very bad. Fig. 18 shows the convergence history for the fully turbulent computation and for the computation with fixed point transition, fig. 19 shows the convergence history for the computations with determined transition locations applying point transition and transition lengths. The computations with determined transition locations were initialized with the flow field from the fully turbulent computation.

Although none of the computations converged within an acceptable number of RANS cycles - this behaviour was expected - the transition determination strategy worked succesfully. The following values for the transition locations and the transition lengths were determined: point transition:

$$
\begin{aligned}
& x_{\text {tr }}^{\text {slat }}=-0.0944, \\
& x_{\text {tr }}^{\text {main }}=0.4175, \\
& x_{\text {tr }}^{\text {flap }}=0.9103,
\end{aligned}
$$

transition lengths:

$$
\begin{aligned}
& x_{\text {tr }}^{\text {slat }}=-0.0944, \Delta s_{t r}{ }_{\text {slat }}=0.0155(7.6 \%), \\
& x_{\text {tr }}^{\text {main }}=0.215, \Delta s_{t r} \text { main }=0.0352(4.3 \%), \\
& x_{t r} \text { flap }=0.9136, \Delta s_{t r} \text { flap }=0.0150(5.0 \%) .
\end{aligned}
$$


The computations using the Wilcox k- $\omega$ model converge after a large number of RANS iteration cycles, fig.20. The fully turbulent calculation needs about 120,000 cycles, the calculation with fixed transition about 70,000 cycles to converge. In the convergence history of the computations with determined transition locations shown in the figure, the convergence history of the fully turbulent forerun is missing. In all the curves of the force coefficients small oscillations remain. The following values for the transition locations and the transition lengths were determined:

point transition:

$$
\begin{aligned}
& x_{t r}^{\text {slat }}=-0.0995, \\
& x_{\text {tr }}^{\text {main }}=0.215, \\
& x_{t r} \text { flap }=0.9056,
\end{aligned}
$$

transition lengths:

$$
\begin{aligned}
& x_{t r}^{\text {slat }}=-0.0995, \Delta s_{t r}{ }_{\text {slat }}=0.0154(7.5 \%), \\
& x_{\text {tr }}^{\text {main }}=0.215, \Delta s_{t r} \text { main }=0.0367(4.5 \%), \\
& x_{t r} \text { flap }=0.9056, \Delta s_{t r} \text { flap }=0.0134(4.5 \%) .
\end{aligned}
$$

The LEA $\mathrm{k}-\omega$ model is even a bit more sensitive than the Wilcox $\mathrm{k}-\omega$ model, fig. 21 . The fully turbulent computation is comparable to the Wilcox $\mathrm{k}-\omega$ model, the computation with fixed point transition, however, could not be initialized with the flow field from the fully turbulent computation. In this case, the computation ended up with negative pressures and densities in some parts of the computational domain and the FLOWer code stopped. Here, the converged solution from the computation with fixed point transition using the Wilcox k- $\omega$ model was used to initialize the flow field. For the computations with determined transition locations the standard procedure could be applied. In the convergence history of the computations with determined transition locations shown in the figure, the convergence history of the fully turbulent forerun is missing. In all the curves of the force coefficients small oscillations remain. The following values for the transition locations and the transition lengths were determined:

point transition:

$$
\begin{aligned}
& x_{t r}{ }_{\text {slat }}=-0.0995, \\
& x_{t r} \text { main }=0.215, \\
& x_{t r}{ }_{\text {flap }}=0.9035,
\end{aligned}
$$

transition lengths:

$$
\begin{aligned}
& x_{t r}^{\text {slat }}=-0.0995, \Delta s_{t r}{ }^{\text {slat }}=0.01536(7.5 \%), \\
& x_{t r}{ }_{\text {main }}=0.215, \Delta s_{t r} \text { main }=0.03613(4.4 \%), \\
& x_{t r} \text { flap }=0.9035, \Delta s_{t r} \text { flap }=0.01323(4.4 \%) .
\end{aligned}
$$

In both cases, a small transitional separation region remains on the upper side of the flap. The separation region begins at $x_{\text {sep }}$ flap $=0.9056$ and has an extension of three grid points on the surface.

\subsection{Conclusions}

The algorithms for the detection of laminar separation works automatically for multi-element configuration on structured multi-block meshes. Its application is independent of the grid structure and the grid topology.

The basic tests for which the functionality of the algorithms and the transition determination strategy was tested were performed using the Spalart-Allmaras turbulence model with Edwards modification. The separation points were succesfully set as transition points and the determination procedure converges in about 10 to $25 \%$ of the RANS cycles that are needed to obtain a fully converged steady solution of the flow field.

The determination procedure works succesfully applying point transition and two different transition length models, the one based on boundary layer properties, formula (a), the other one, formula (b), based on flat plate theory. Formula (a) yields transition lengths which are much greater compared to experimental findings and small transitional separation regions on some of the elements of the configuration directly downstream of the transition locations. Formula (b) yields transition lengths which have a value of about $5 \%$ of the chord length of the corresponding element which is a value that suits to the experiments. The transitional separation regions do not appear.

In addition to the Spalart-Allmaras model with Edwards modification the algebraic Baldwin-Lomax model, the standard Wilcox k- $\omega$ model and the LEA k- $\omega$ model have been applied. All the computations using these other turbulence models had to be carried out in single grid mode as for multigrid computations no convergence could be obtained.

The transition determination procedure worked succesfully in all cases, also in the cases using the BaldwinLomax turbulence model that did not converge with respect to the RANS iteration, which was not expected. All determined values for the transition locations and the transition lengths are of the expected magnitude.

The next steps to be done, are the coupling of the extended FLOWer code to the transition prediction module and the comparison of the results with experimental data for the selected test case.

\section{Acknowledgement}

This work has been carried out within the HiAer Project (High Level Modelling of High Lift Aerodynamics). The HiAer project is a collaboration between DLR, ONERA, KTH, HUT, TUB, Alenia, EADS Airbus, QinetiQ and FOI. The project is managed by FOI and is partly funded by the European Union (Project Ref: G4RD-CT-2001-00448).

We would like to acknowledge BAE Systems for letting us use the A310 59\% section data in this study.

\section{References}

${ }^{1}$ Radespiel, R.; Graage, K.; Brodersen, O.: " Transition Predictions Using Reynolds-Averaged Navier-Stokes 
and Linear Stability Analysis Methods “, AIAA 91-1641, 1991

${ }^{2}$ Smith, A.M.O.; Gamberoni, N.: "Transition, Pressure Gradient and Stability Theory, " Douglas Aircraft Company, Long Beach , Calif. Rep. ES 26388, 1956

${ }^{3}$ van Ingen, J.L.: " A suggested Semi-Empirical Method for the Calcula- tion of the Boundary Layer Transition Region," Univ. of Delft, Dept. Aerosp.Eng., Delft, The Netherlands, Rep. VTH-74, 1956

${ }^{4}$ Stock, H. W.; Haase, W.: "A Feasibility Study of $\mathrm{e}^{N}$ Transition Prediction in Navier-Stokes Methods for Airfoils“", AIAA Journal, Vol.37, no. 10, pp. 1187-1196, 1999

[5] Horton, H. P.; Stock, H. W.: " Computation of Compressible, Laminar Boundary Layers on Swept, Tapered Wings“", J. Aircraft, Vol.32, pp.1402-1405, 1995

[6] Stock, H. W.; Degenhardt, E.: “A simplified $\mathrm{e}^{N}$ method for transition prediction in two-dimensional, incompressible boundary layers", Z. Flugwiss. Weltraumforsch., Vol.13, pp.16-30, 1989

[7] Warren, E. S.; Hassan, H. A.: "Transition Closure Model for Predicting Transition Onset" ", J. Aircraft, Vol.35, pp. 769-775, 1998

[8] Czerwiec, R.M.; Edwards, J. R.; Rumsey, C. L.; Bertelrud, A.; Hassan, H. A.: "'Study of High-Lift Configurations Using k-z Transition/Turbulence Model ", AIAA 99-3186, 1999

${ }^{9}$ Edwards, J. R.; Roy, C. J.; Blottner, F. G.; Hassan, H. A.: "Development of a One-Equation Transition/Turbulence Model“", AIAA Journal, Vol.39, no. 9, pp. 16911698, 2001

${ }^{10}$ FLOWer - Installation and User Handbook, Release 116, Doc.Nr. MEGAFLOW-1001, Institut für Entwurfsaerodynamik, Deutsches Zentrum für Luft- und Raumfahrt e.V., 2000

${ }^{11}$ Krumbein, A.; Stock, H. W.: "Laminar-turbulent Transition Modeling in Navier-Stokes Solvers using Engineering Methods" ", ECCOMAS 2000, Barcelona (e), 11.-14. September 2000, ECCOMAS 2000 - CDRom Proceedings, Hrsg.: International Center for Numerical Methods in Engineering (CIMNE), 2000, ISBN: 84-89925-70-4, Depósito Legal: B-37139-2000

${ }^{12}$ Krumbein, A.: "Coupling of the DLR Navier-Stokes Solver FLOWer with an $\mathrm{e}^{N}$-Database Method for laminar-turbulent Transition Prediction on Airfoils" ", Notes on Numerical Fluid Mechanics-Vol.77, pp. 92-99, Springer Verlag, 2002

${ }^{13}$ Stock, H. W.; Haase, W.: "Navier-Stokes Airfoil Computations with $\mathrm{e}^{N}$ Transition Prediction Including Transitional Flow Regions" ", AIAA Journal, Vol.38, no. 11, pp. 2059-2066, 2000

${ }^{14}$ High Level Modelling of High Lift AerodynamicsHiAer, Annex 1, "Description of Work", GROWTH Project GRD1-2000-25226 HiAer
${ }^{15}$ Dhawan, S.; Narasimha, R.: "Some properties of boundary layer flow during the transition from laminar to turbulent motion, " Journal of Fluid Mechanics, Vol.3, pp. 418-436, 1958

${ }^{16}$ Walker, G. J.: " Transitional Flow on Axial Turbomachine Blading “, AIAA Journal, Vol.27, No. 5, pp. 595602, 1989

${ }^{17}$ Brodersen, O.; Ronzheimer, A.; Ziegler, R.; Kunert, T.; Wild, J.; Hepperle, M.: “'Aerodynamic Applications using MegaCads", Proc. of 6th International Conference on Numerical Grid Generation in Computational Field Simulation, editor: M. Cross, publisher: ISGG, NSF Eng. Research Center, Mississippi State University, pp. 793-802, 1998

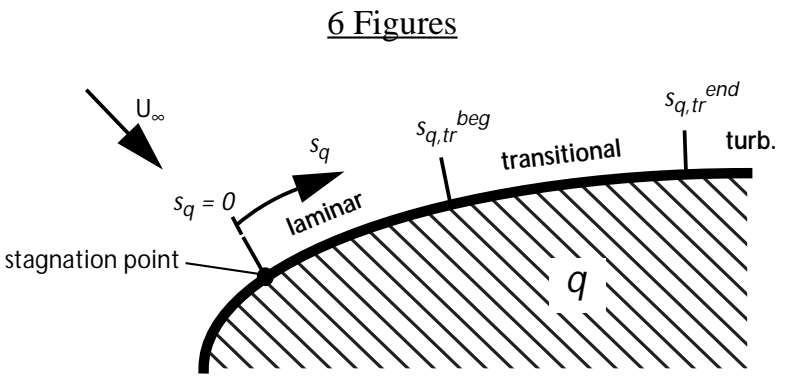

Fig.1: stylised depiction of laminar, transitional and turbulent flow regions at surface side $q$

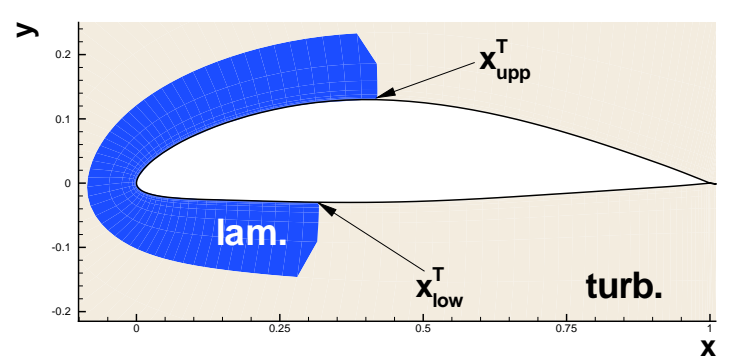

Fig.2: laminar zone in a turbulent rest of the flow domain - point transition

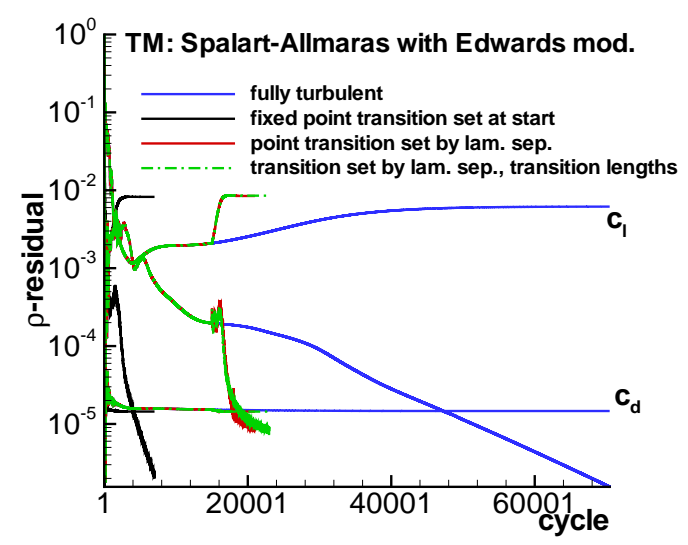

Fig.3: convergence history of RANS computations for the test case 
separation points (cycles): point transition

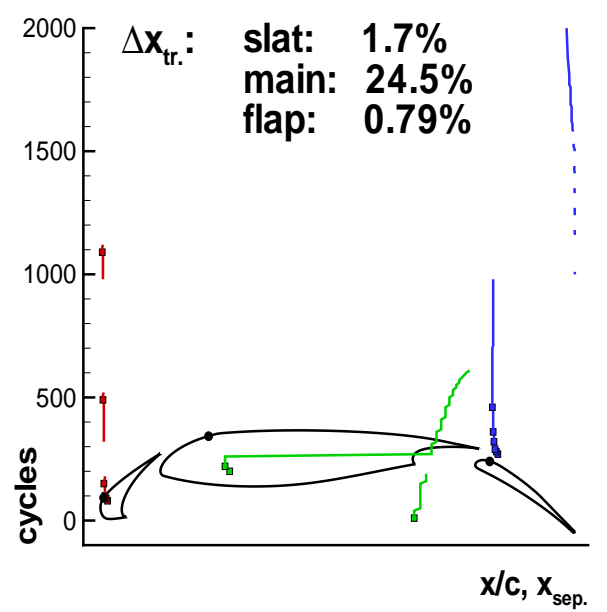

Fig.4: convergence history of transition locations, point transition

separation points (cycles): point transition

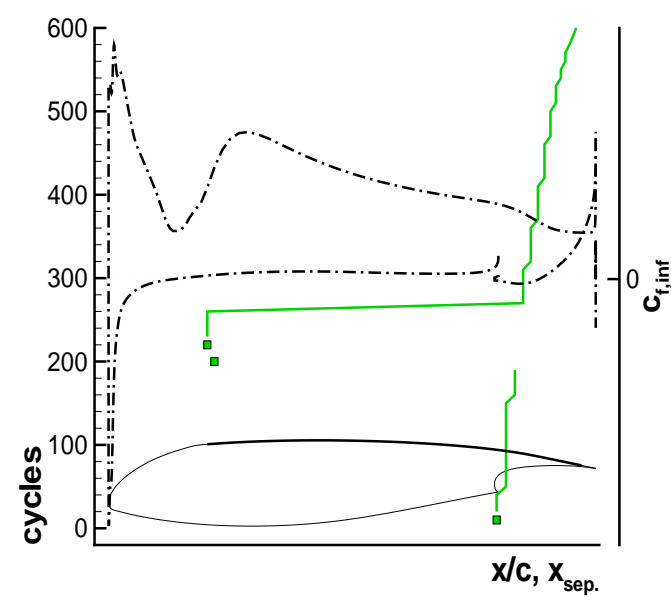

Fig.6: transition locations and $c_{f}$-distribution on main airfoil, point transition

separation points (cycles): point transition

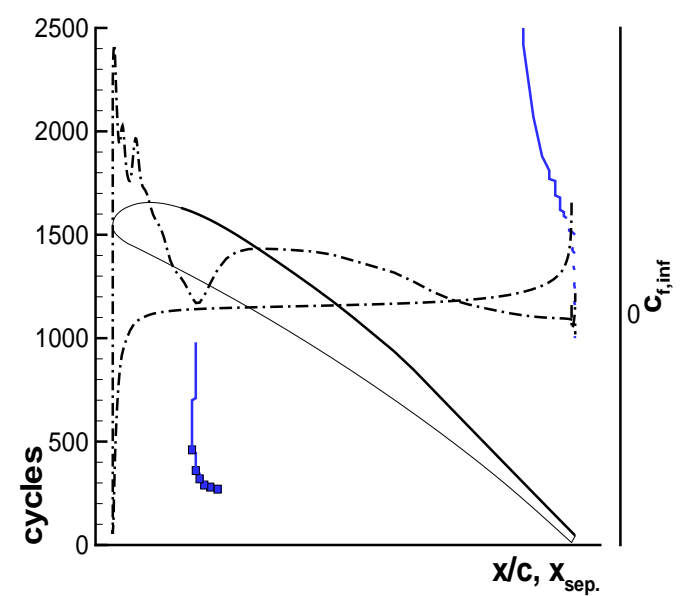

Fig.8: transition locations and $c_{f}$-distribution on flap, point transition separation points (cycles): transition lengths (a)

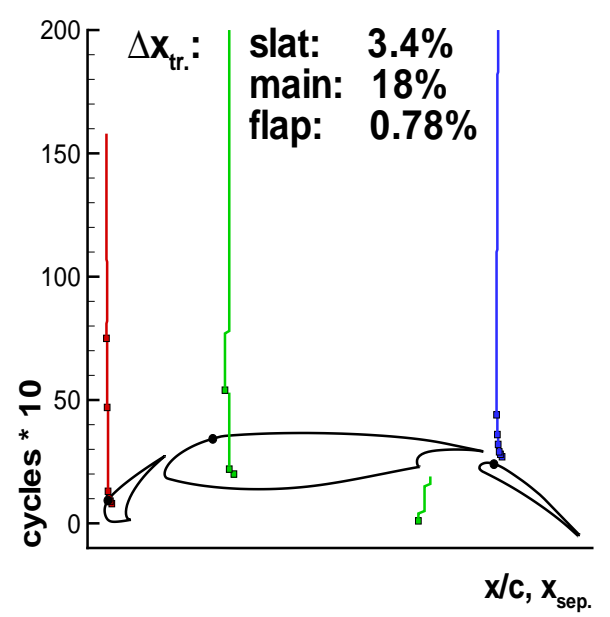

Fig.5: convergence history of transition locations, transition lengths, formula (a)

separation points (cycles): transition lengths (a)

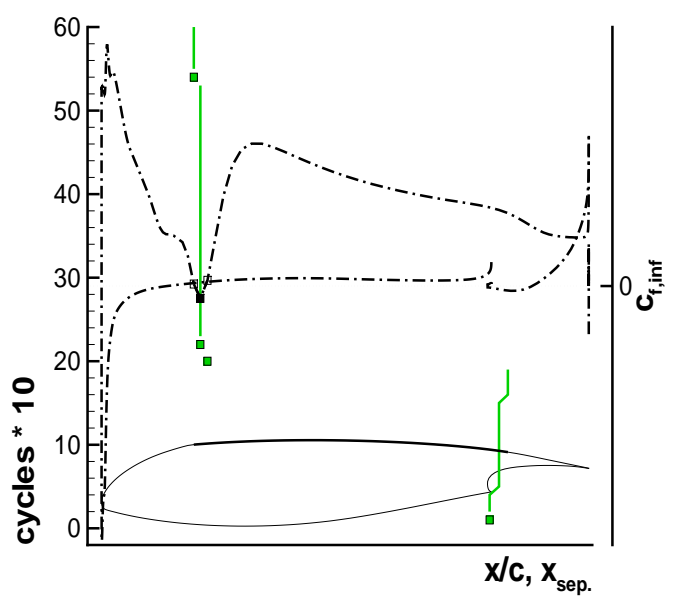

Fig.7: transition locations and $c_{f}$-distribution on main airfoil, transition lengths, formula (a)

separation points (cycles): transition lengths (a)

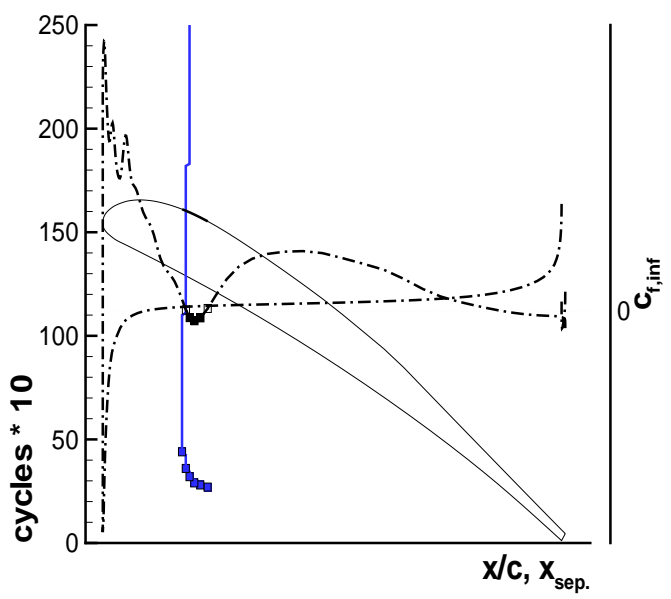

Fig.9: transition locations and $c_{f}$-distribution on flap, transition lengths, formula (a) 


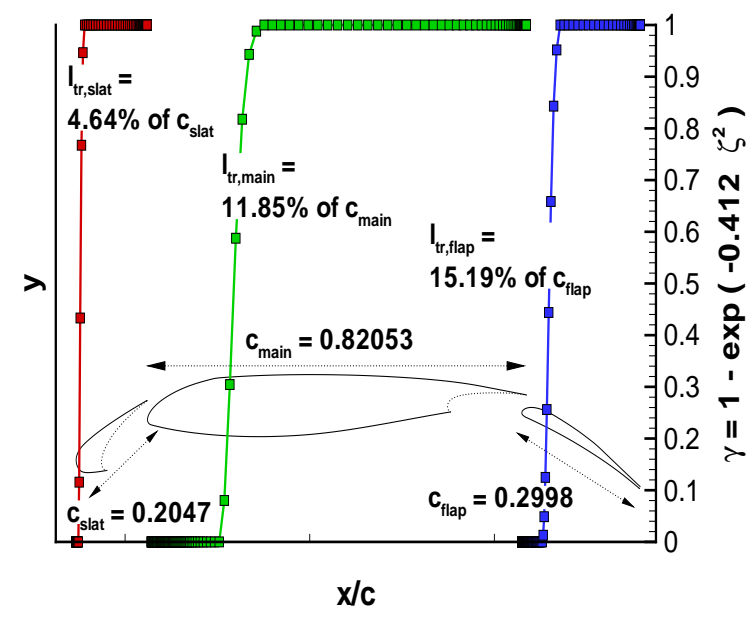

Fig.10: transition lengths, formula (a)

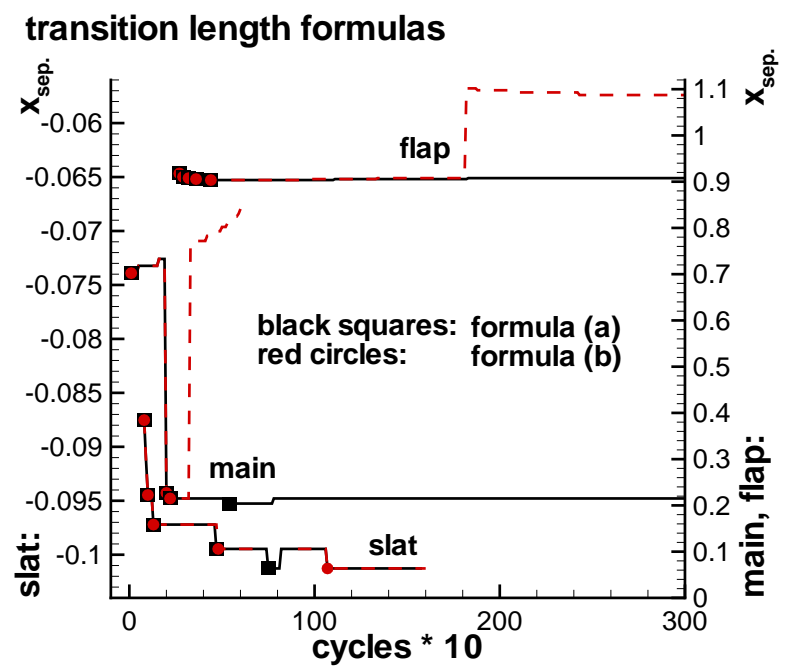

Fig.12: convergence history of transition locations, formula (a) vs. formula (b)

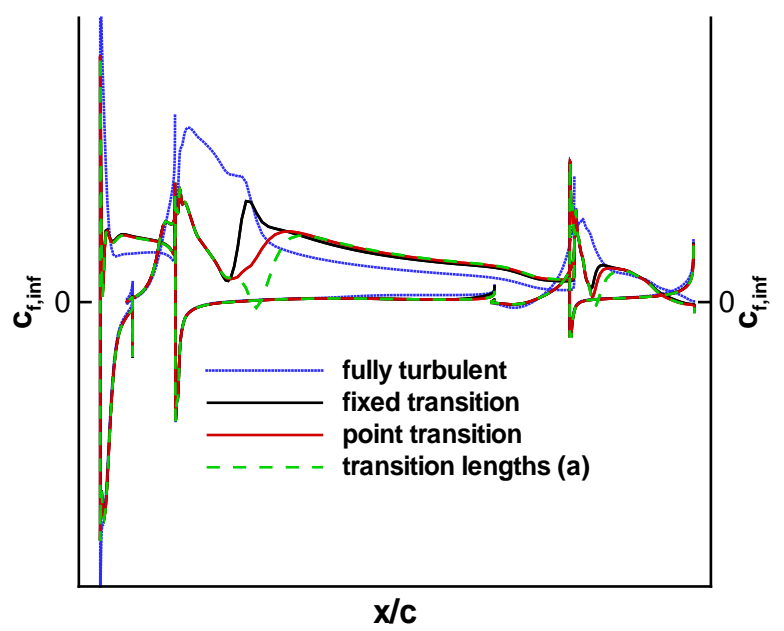

Fig.14: $c_{f}$-distributions, fully turbulent, prescribed and determined point transition, determined transition formula (a)

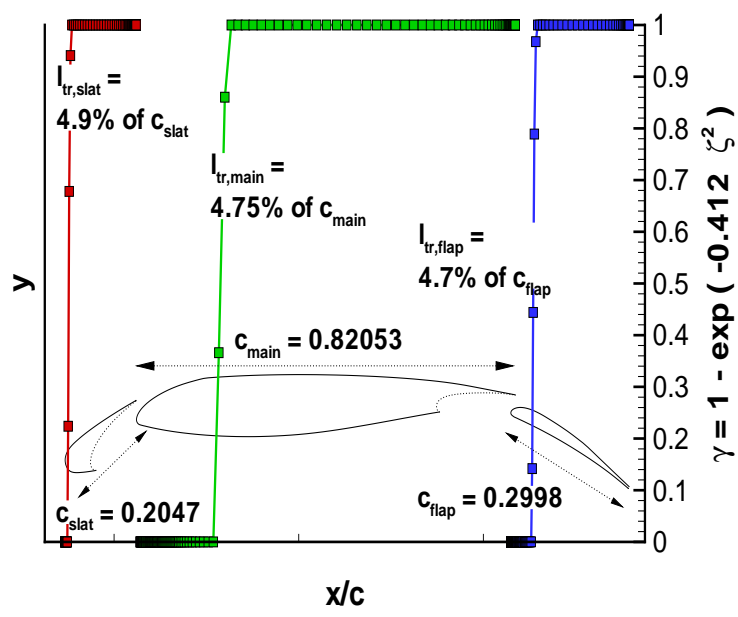

Fig.11: transition lengths, formula (b)

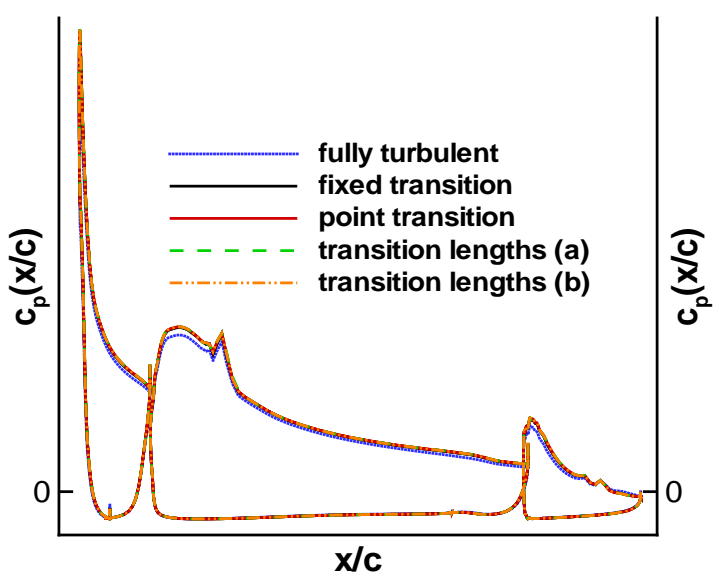

Fig.13: $c_{p}$-distributions

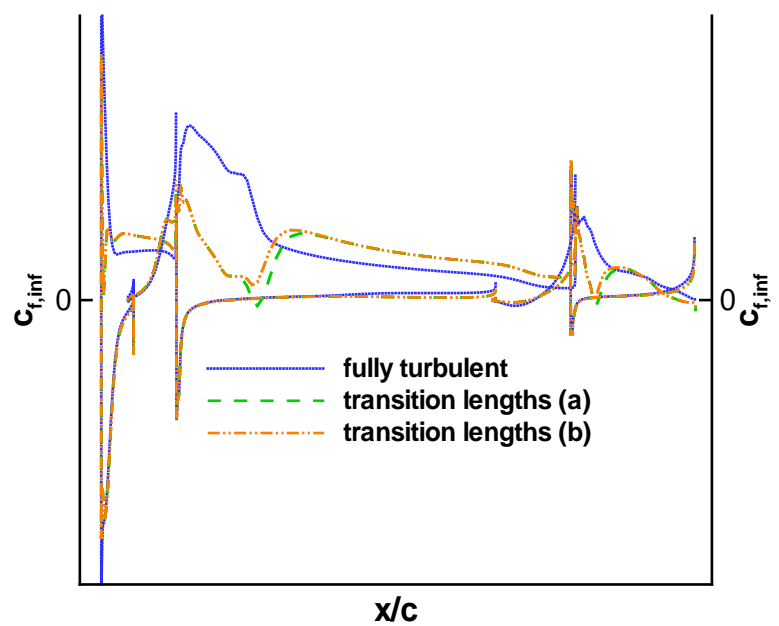

Fig.15: $c_{f}$-distributions, determined transition, formula (a) vs. formula (b) 
separation points (cycles): point transition without fully turbulent initialization

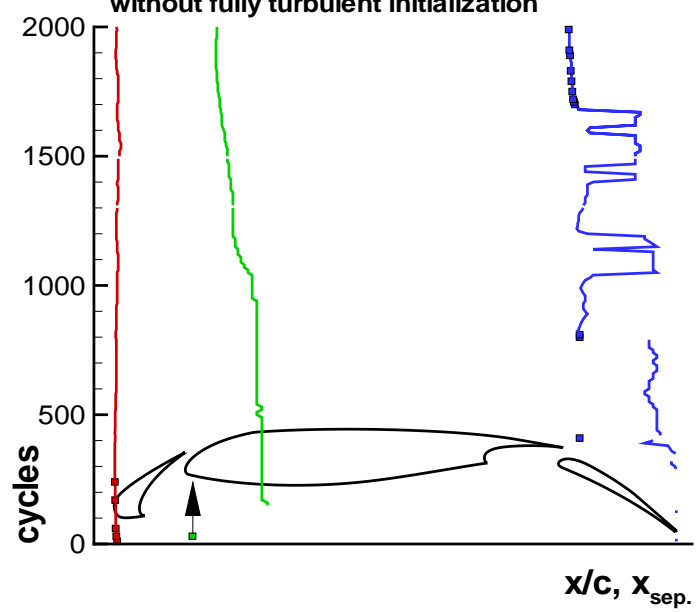

Fig.16: convergence history of transition locations, point transition, without fully turbulent initialization of flow field separation points (cycles): transition lengths (a)

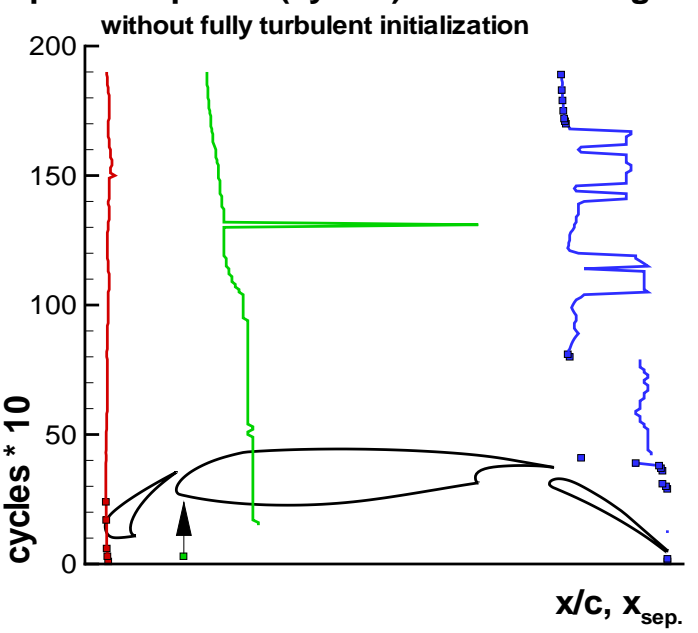

Fig.17: convergence history of transition locations, transition lengths, formula (a), without fully turbulent initialization of flow field

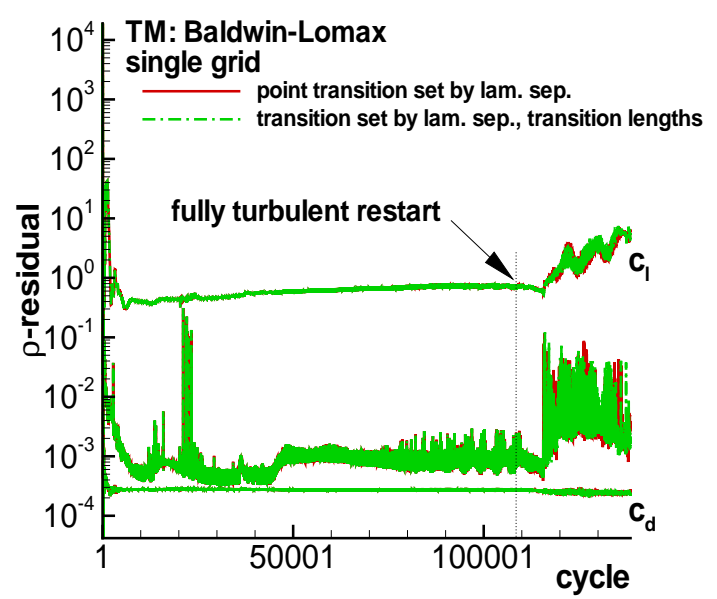

Fig.19: convergence history of RANS computations using the BaldwinLomax turbulence model, predicted transition, point transition and transition lengths

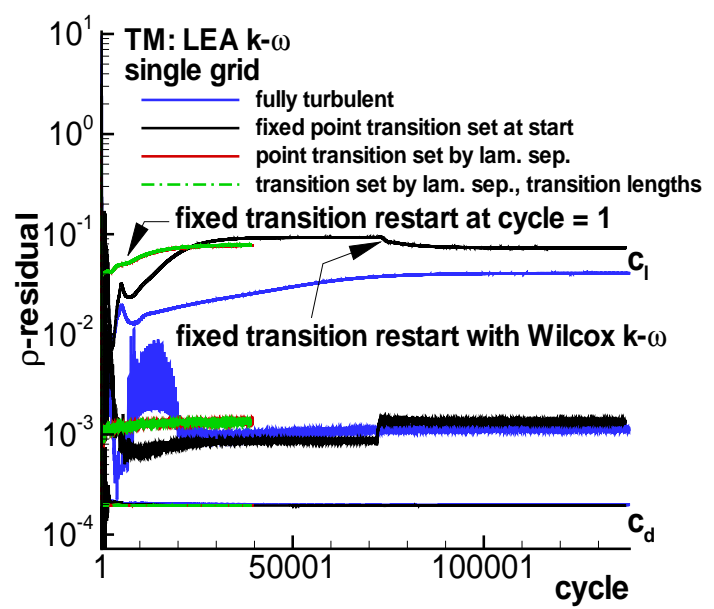

Fig.21: convergence history of RANS computations using the LEA k- $\omega$ model 